[8] T. P. Chen, S. Li, S. Fung, and K. F. Lo, "Interface trap generation by FN injection under dynamic oxide field stress," IEEE Trans. Electron Devices, vol. 45, pp. 1920-1926, Sept. 1998.

[9] Y. Hokari, "Stress voltage polarity dependence of thermally grown thin gate oxide wearout," IEEE Trans. Electron Devices, vol. 35, pp. 1299-1304, Aug. 1988.

[10] D. J. Dumin, S. K. Mopuri, S. Vanchinathan, R. S. Scott, R. Subramoniam, and T. G. Lewis, "High field related thin oxide wearout and breakdown," IEEE Trans. Electron Devices, vol. 42, pp. 760-772, Apr. 1995.

[11] T. Wang, L.-P. Chiang, N.-K. Zous, C.-F. Hsu, L.-Y. Huang, and T.-S Chao, "A comprehensive study of hot carrier stress-induced drain leakage current degradation in thin-oxide n-MOSFETs," IEEE Trans. Electron Devices, vol. 46, pp. 1877-1882, Sept. 1999.

\section{Determination of Channel Temperature in AIGaN/GaN HEMTs Grown on Sapphire and Silicon Substrates Using DC Characterization Method}

J. Kuzmík, P. Javorka, A. Alam, M. Marso, M. Heuken, and P. Kordoš

\begin{abstract}
Self-heating effects and temperature rise in AlGaN/GaN HEMTs grown on silicon and sapphire substrates are studied, exploiting transistor dc characterization methods. A negative differential output resistance is observed for high dissipated power levels. An analytical formula for a source-drain current drop as a function of parasitic source resistance and threshold voltage changes is proposed to explain this behavior. The transistor source resistance and threshold voltage is determined experimentally at different elevated temperatures to construct channel temperature versus dissipated power transfer characteristic. It is found that the HEMT channel temperature increases rapidly with dissipated power and at $6 \mathrm{~W} / \mathrm{mm}$ reaches values of $\sim 320^{\circ} \mathrm{C}$ for sapphire and $\sim 95^{\circ} \mathrm{C}$ for silicon substrate, respectively.
\end{abstract}

Index Terms-III-nitrides, power FETs, semiconductor device measurements, semiconductor device thermal factors.

\section{INTRODUCTION}

III-nitride-based devices are exceptionally suitable for high-frequency, power, and temperature applications. Microwave performance of $\mathrm{AlGaN} / \mathrm{GaN}$ HEMT at the ambient temperature of $300^{\circ} \mathrm{C}$ was already documented [1]. However, the HEMT performance deterioration reflected by transconductance and power added efficiency depression could be observed at elevated temperature [2]. The same is valid for self-heating phenomenon when the HEMT channel is heated by dissipated power [3], [4]. Therefore, for device optimization, it is important to study the self-heating effects in AlGaN/GaN HEMTs at various operation conditions, for various substrate materials (on which the device is grown) and for different device geometry. Recently, an innovative technological approach has been introduced where conventional sapphire substrate is replaced by a silicon one [5], [6]. Aside from reducing device costs, a higher thermal conductance of

Manuscript received May 13, 2002. This work was supported by the Slovak Grant Agency for Science Grant 2/7200/21. The review of this brief was arranged by Editor A. Medhi.

J. Kuzmík is with the Institute of Electrical Engineering Slovak Academy of Sciences, 84239 Bratislava, Slovakia. (e-mail: elekkuzm@ @avba.sk).

P. Javorka, M. Marso, and P. Kordoš are with the Institute of Thin Films and Interfaces (ISG-1), Research Centre Jülich, D-52425 Jülich, Germany.

A. Alam and M. Heuken are with Aixtron AG, D-52072 Aachen, Germany.

Publisher Item Identifier 10.1109/TED.2002.801430. silicon promises better device cooling and brings a prospect of a higher power performance.

Thermal resistance (temperature rise per Watt) of $\mathrm{AlGaN} / \mathrm{GaN}$ HEMTs grown on sapphire has been already experimentally determined [4]. However, the channel temperature was assumed to be equal to the temperature of the external heater. Recently, Raman spectroscopy has been used for an evaluation of the channel temperature [7]. Special test structures with large separation between the contacts are needed in that case. In this work we present dc characterization method of the channel temperature determination which can be easily applied directly on any device layout down to submicron dimensions. We compare temperature rise in $\mathrm{AlGaN} / \mathrm{GaN}$ HEMTs grown on sapphire and silicon substrates. The method is also used on structures with a different layout to investigate the role of the transistor gate dimensions.

\section{EFFECt OF Self-Heating ON THE TRANSISTOR DC PERFORMANCE}

Measurements of the HEMT output characteristics and the transistor transconductance $g_{m}$ are basic tools for the HEMT dc performance evaluation. Here, parasitic source resistance $R_{S}$ together with values of the sheet carrier concentration and the electron saturation velocity $v_{\text {sat }}$ are decisive. $R_{S}$ of the transistor comprises of two components: the contact resistance and the resistance of the channel between the source and gate contacts. If the device temperature is raised, the low-field electron mobility in the channel is decreased [8], and a proportional increase of the channel resistance related to the source resistance $R_{S}$ can be expected. The voltage drop on $R_{S}$ modifies the source-gate potential in a way that the conductive path for electrons is being pinched off. Consequently, the source-drain saturation current $I_{\text {sat }}$ is reduced by the value of $\sim I_{\text {sat }} g_{m} \Delta R_{S}$.

On the other hand, electron velocity will saturate in the channel under the gate. Monte Carlo simulation have proved that $v_{\text {sat }}$ decreases with the temperature [9], [10]. Similarly, HEMT threshold voltage change $\Delta V_{T}$ (given by the sheet carrier concentration and by the Schottky contact barrier height) must be considered. Thus the $I_{\text {sat }}$ drop due to the intrinsic HEMT parameters variation can be expressed as $\sim g_{m} \Delta V_{T}-I_{\text {sat }} \Delta v_{\text {sat }} / v_{\text {sat }}$, where $\Delta v_{\text {sat }}$ is the velocity change in respect to the reference value (at room temperature).

The transistor dissipated power determines the channel temperature $T$ and thus in the case of transistor output characteristics, $R_{S}, v_{\text {sat }}$, and $V_{T}$ values are becoming a function of the drain voltage. Therefore, for high power levels and low substrate leakage, the dc output characteristics may show a substantial drop in the saturation current with increased drain voltage and a negative differential resistance may appear. We define $\Delta I_{\text {sat }}$ as a difference $I_{\text {sat }}-I_{\text {sat }}^{0}$, where $I_{\text {sat }}$ is a value in a particular HEMT working point and $I_{\text {sat }}^{0}$ denounced a reference value of the channel at room temperature ( $I_{\mathrm{sat}}^{0}$ is obtained by extrapolating $I_{\mathrm{sat}}$ to $V_{D}=0 \mathrm{~V}$, see Fig. 1). If $\Delta R_{S}\left(\Delta v_{\text {sat }}, \Delta V_{T}\right)$ is analogously defined, i.e., $\Delta R_{S}\left(\Delta v_{\text {sat }}, \Delta V_{T}\right)$ is given by $R_{S}\left(v_{\text {sat }}, V_{T}\right)$ increase (with dissipated power) from its room temperature value, we can write

$$
\Delta I_{\text {sat }}=-g_{m}\left(I_{\text {sat }} \Delta R_{S}+\Delta V_{T}\right)+\frac{I_{\text {sat }} \Delta v_{\text {sat }}}{v_{\text {sat }}}+\frac{V_{D}}{R_{\text {sub }}}
$$

where $V_{D} / R_{\mathrm{sub}}$ represents the leakage current through the substrate. The $R_{\text {sub }}$ can be determined from the transistor output resistance by low power levels when self-heating is negligible, i.e., for $V_{G}$ close to the pinch-off.

If functional dependencies $R_{S}=f(T), V_{T}=f(T)$, and $v_{\text {sat }}=f(T)$ are experimentally determined (using independent measurement), transistor output characteristics can be used to construct 


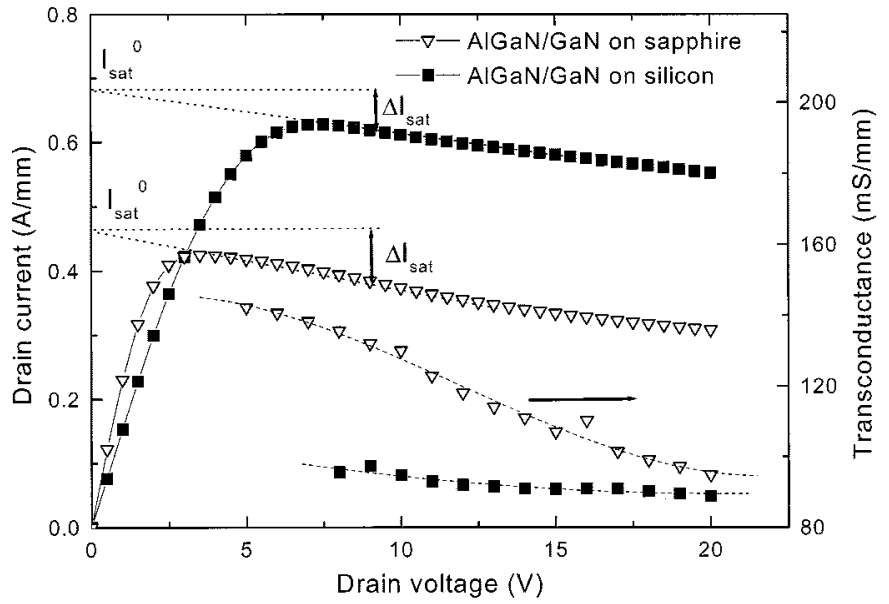

Fig. 1. AlGaN/GaN HEMTs output and transconductance characteristics al $V_{G}=0 \mathrm{~V}$.

channel temperature versus dissipated power transfer characteristics. It was suggested to use cutoff frequency values at different temperatures to get the $v_{\text {sat }}=f(T)$ dependence [11]. However, besides of ignoring the role of the $R_{S}$, the cited method does not consider the channel self-heating. On the other hand theoretical calculations [9], [10] indicate that $v_{\text {sat }}$ in $\mathrm{GaN}$ is much less sensitive to temperature as in GaAs. Moreover, strong polarization fields in AlGaN/GaN quantum well further eliminate electron mobility dependence on temperature [12]. Therefore, in our work we consider $v_{\text {sat }}$ to be constant and the possible error of this assumption is discussed at the end of the experiment.

The HEMT maximum output rf power capability relates to dc parameters as $P_{\mathrm{rf}}=(\Delta V \times \Delta I) / 8$. Here the maximum voltage swing $\Delta V$ is given by a difference of a maximum source-drain break-down voltage and a minimum knee voltage. The maximum current swing $\Delta I$ is given by a maximum source-drain saturation current. This further emphasizes the relevance of the HEMT dc characterization.

\section{EXPERIMENT}

The AlGaN/GaN HEMT structures used in this study were grown on 330- $\mu \mathrm{m}$-thick sapphire or silicon wafers by MO-VPE. Sapphire-based structures consist of a $3-\mu \mathrm{m}$-thick GaN buffer, and a 20 -nm $\mathrm{Al}_{0.2} \mathrm{Ga}_{0.8} \mathrm{~N}$. The epi-layers were nominally undoped, using the polarization effect to generate free carriers in the quantum well. In the case of a silicon substrate a 6-nm undoped AlGaN spacer $(23 \%$ $\mathrm{Al}$ content), a 20-nm Si-doped AlGaN carrier-supply and a 6-nm undoped $\mathrm{AlGaN}$ barrier layer were grown on top of a GaN buffer. The devices were prepared by a conventional device processing procedure applying combination of e-beam (the gate length of $0.25,0.45$, and $0.65 \mu \mathrm{m}$ ), optical lithography and a dry etching for a mesa definition. The ohmic contacts consist of $\mathrm{Ti} / \mathrm{Al} / \mathrm{Ni} / \mathrm{Au}$ and they were annealed at $900^{\circ} \mathrm{C}$, while $\mathrm{Ni} / \mathrm{Au}$ was used for a Schottky contact formation.

The dc output characteristics of both types of AlGaN/GaN HEMTs with $50-\mu \mathrm{m}$ gate width and $0.45 \mu \mathrm{m}$ gate length were investigated initially. The current-voltage $(I-V)$ characteristics at higher dissipated power levels (gate grounded) show clear drop $\Delta I_{\text {sat }}$ in the saturation current (Fig. 1). Transconductance characteristics shown in Fig. 1 also prove transconductance depression with drain voltage. More steep dependence $g_{m}=f\left(V_{D}\right)$ for device grown on sapphire has been observed. We note that trapped charge may deplete the HEMT channel for a subsequent $I_{\text {sat }}$ traces [13]. Therefore, before any subsequent measurement the device charge must be restored (e.g., by illumination or

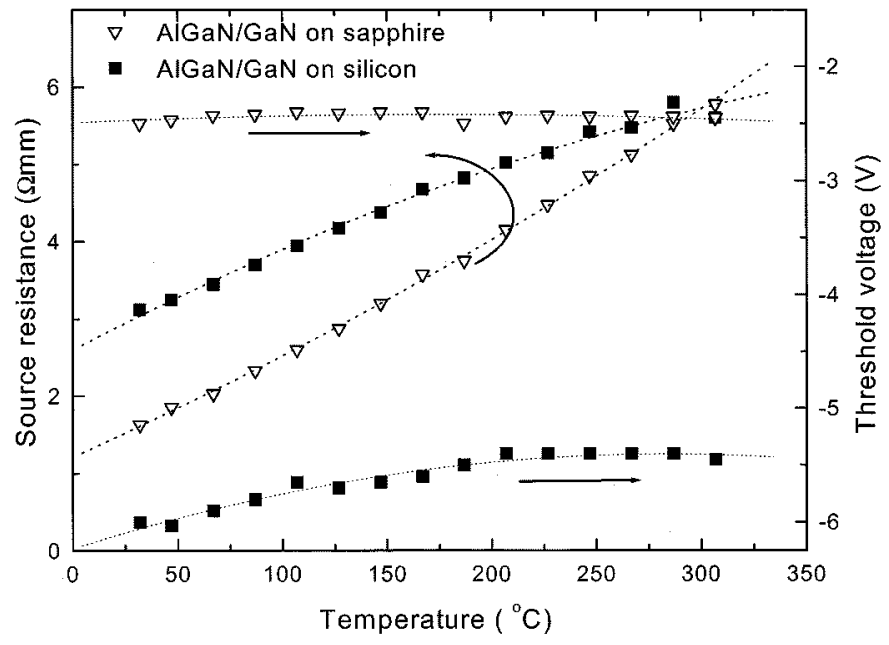

Fig. 2. Dependencies of the AlGaN/GaN HEMTs source resistance and threshold voltage on ambient temperature.

by the positive voltage pulse on the gate) [14], and only first $I-V$ trace can be evaluated.

Next we measured the HEMT source resistance $R_{S}$ and threshold voltage $V_{T}$ at different ambient temperatures $T$ (Fig. 2) using external heater. The $R_{S}$ has been extrapolated from the functional dependence of the source-drain resistance divided by 2 in a linear regime $\left(V_{D}=0.5 \mathrm{~V}\right)$ [15]. To eliminate uncertainty caused by the carrier injection and by short channel effects [16], linear regime was also used by the $V_{T}$ determination. Because of low dissipated power $(\sim 50 \mathrm{~mW} / \mathrm{mm})$ during these measurement, the channel temperature increase due to the self-heating effect could be neglected. The $R_{S}$ increases from $\sim 1.6 \Omega \mathrm{mm}$ and $3.1 \Omega \mathrm{mm}$ for sapphire and silicon-based HEMTs at room temperature, respectively, to $\sim 5.7 \Omega \mathrm{mm}$ at $310^{\circ} \mathrm{C}$ where both dependencies surprisingly meet. We assume that different HEMT doping mechanisms (modulation doping of HEMT on silicon versus polarization doping of HEMT on sapphire), together with different processing procedures determines different electron scattering mechanisms in the channel which account for observed different thermal dependencies. Fig. 2 also shows that structures grown on sapphire exhibit almost constant values of $V_{T}$ while clear $V_{T}$ increase with temperature was observed for AlGaN/GaN HEMTs on silicon. Taking into account the marginal change of the Schottky barrier height (not shown), the total sheet carrier density seems to decrease with increased temperature for this type of the structure. The explanation for this is not known. However, similar phenomena was also reported for the HEMT grown on SiC [17]. The carrier density decrease with the increased temperature in the HEMT grown on silicon may also account for less pronounce increase of $R_{S}$ with $T$ (see Fig. 2) in this type of the device.

Finally, using polynomial fits $R_{S}=f(T)$ and $V_{T}=f(T)$ (Fig. 2), together with the measured $\Delta I_{\text {sat }}$ (at $V_{G}=0 \mathrm{~V}$ ) and transconductance as a function of the drain voltage (Fig. 1), an iterative solution of (1) was performed to obtain channel temperature versus power transfer characteristics (Fig. 3). From the constructed characteristic it can be seen that the channel temperature $T$ increases much more rapidly for HEMT grown on sapphire reaching $320^{\circ} \mathrm{C}$ at $6 \mathrm{~W} / \mathrm{mm}$ dissipated power in comparison to $95^{\circ} \mathrm{C}$ for a silicon substrate HEMT at the same power level. This clearly confirms the advantage of silicon substrate for high power AlGaN/GaN HEMTs.

Additionally, we have also investigated the channel temperature increase with dissipated power of AlGaN/GaN HEMTs grown on sapphire for a different gate length. Results shown in Fig. 4 demonstrate 


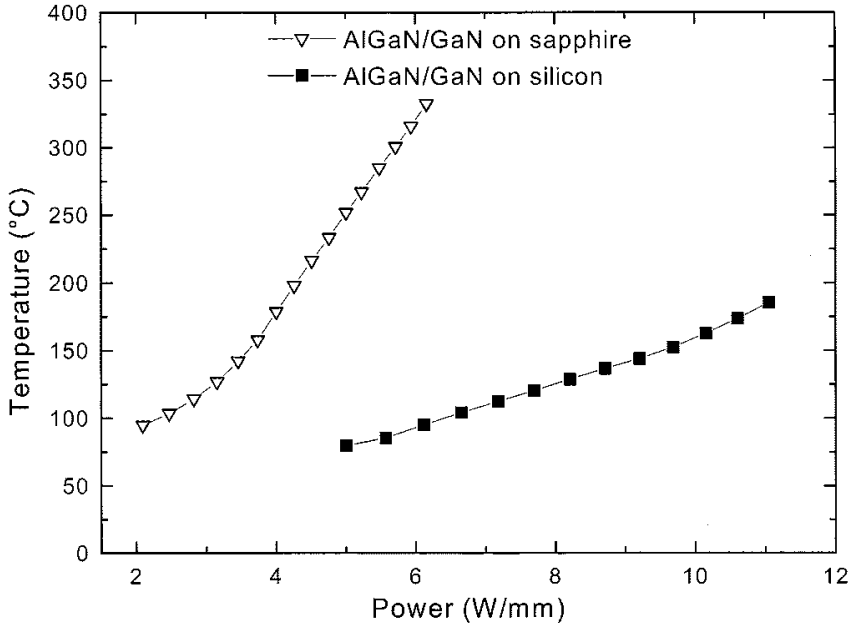

Fig. 3. AlGaN/GaN HEMTs dissipated power-to-channel temperature transfer characteristics for silicon and sapphire substrates.

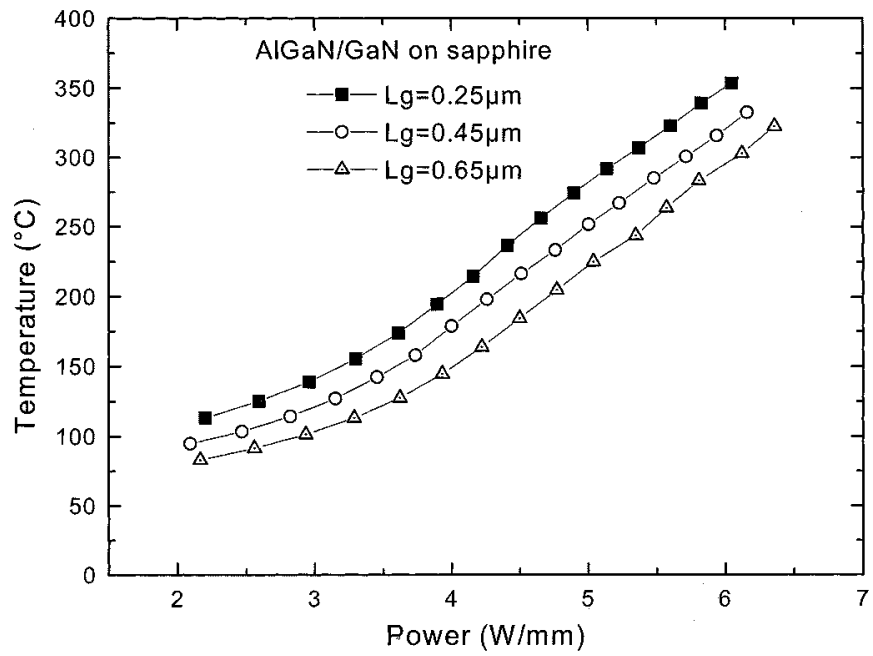

Fig. 4. AlGaN/GaN HEMTs dissipated power-to-hannel temperature transfer characteristics for different gate lengths.

that the gate dimension influence cannot be underestimated $\left(30-50^{\circ} \mathrm{C}\right.$ temperature difference between 0.65 and $0.25-\mu \mathrm{m}$ gate length device) even though this effect is found to be not crucial. The shorter gate length structures confirm more difficult cooling as predicted by an analytical model [18].

The electron saturation velocity was considered to be constant. Monte Carlo simulations show that $v_{\text {sat }}$ in GaN decreases by $\sim 5.6 \%$ if the temperature is raised from 300 to $500 \mathrm{~K}$ [10]. It was also shown that if $\mathrm{GaN}$ forms a heterojunction with $\mathrm{AlGaN}$, the polarization fields in $\mathrm{AlGaN} / \mathrm{GaN}$ quantum well would reduce the carrier mobility dependence on temperature [12]. We expect (but in lower extent) similar phenomena also for $v_{\text {sat }}$. Considering this and taking into account measured device parameters in (1), we assume that our temperature values might be overestimated by only $\sim 10 \%$. Nevertheless, described method represents a simple way of the channel temperature determination in HEMT, which can be used for a qualitative analysis and applied directly on any device layout.

\section{CONCLUSIONS}

We studied self-heating effects in the AlGaN/GaN HEMTs grown on the sapphire and silicon substrates, respectively. A new simple method of the channel temperature determination is proposed and experimentally verified. We observed that the negative differential resistance in the output characteristics accompanies the HEMT self-heating effect. We have confirmed that devices grown on silicon are better cooled and the AlGaN/GaN HEMT on silicon shows $\sim 4 \times$ lower thermal resistance than the device on sapphire. Finally, studying HEMTs with different gate length, we have verified that the self-heating effect is enhanced for structures with shorter gates.

\section{ACKNOWLEDGMENT}

The first author wishes to thank BMBF for its support.

\section{REFERENCES}

[1] M. A. Khan, M. S. Shur, J. N. Kuznia, Q. Chen, J. Burm, and W. Schaff, "Temperature activated conductance in GaN/AlGaN heterostructure field effect transistors operating at temperatures up to $300^{\circ} \mathrm{C}, " \mathrm{Appl}$. Phys. Lett., vol. 66, pp. 1083-1085, 1995.

[2] M. W. Shin and R. J. Trew, "GaN MESFETs for high-power and high-temperature microwave applications," Electron. Lett., vol. 31, pp. 498-500, 1995.

[3] Y.-F. Wu, B. P. Keller, S. Keller, D. Kapolnek, S. P. Denbaars, and U. K. Mishra, "Measured microwave power performance of AlGaN/GaN MODFET," IEEE Electron Device Lett., vol. 17, pp. 455-457, Sept. 1996.

[4] R. Gaska, A. Osinsky, J. W. Yang, and M. S. Shur, "Self-Heating in high-power AlGaN-GaN HFETs," IEEE Electron Devices Lett., vol. 19, pp. 89-91, Feb. 1998.

[5] E. M. Chumbes, A. T. Schremer, J. A. Smart, Y. Wang, N. C. MacDonald, D. Hogue, J. J. Komiak, S. J. Lichwalla, R. E. Leoni, and J. R. Shealy, "AlGaN/GaN high electron mobility transistors on Si (111) substrates," IEEE Trans. Electron Devices, vol. 48, pp. 420-425, Mar. 2001.

[6] P. Javorka, A. Alam, M. Wolter, A. Fox, M. Marso, M. Heuken, H. Lüth, and P. Kordoš, "AlGaN/GaN HEMTs on (111) silicon substrates," IEEE Electron Device Lett., vol. 23, pp. 4-6, Jan. 2002.

[7] M. Kuball, J. M. Hayes, M. J. Uren, T. Martin, J. C. H. Birbeck, R. S Balmer, and B. T. Hughes, "Measurement of temperature in active highpower AlGaN/GaN heterostructure field effect transistors using Raman spectroscopy," IEEE Electron Device Lett., vol. 23, pp. 7-9, Jan. 2002.

[8] J. D. Albrecht, R. P. Wang, R. P. Ruden, M. Farahmand, and K. F. Brennan, "Electron transport characteristics of $\mathrm{GaN}$ for high temperature device modeling," J. Appl. Phys., vol. 83, pp. 4777-4781, 1998.

[9] M. A. Khan, Q. Chen, M. S. Shur, B. T. Dermott, J. A. Higgins, J. Burm, W. J. Schaff, and L. F. Eastman, "GaN based heterostructure for high power devices," Solid-State Electron., vol. 41, pp. 1555-1559, 1997.

[10] A. F. M. Anwar, S. Wu, and R. T. Webster, "Temperature dependent transport properties in $\mathrm{GaN}, \mathrm{Al}_{x} \mathrm{Ga}_{1-x} \mathrm{~N}$ and $\mathrm{In}_{2 x} \mathrm{Ga}_{1-x_{2}} \mathrm{~N}$ semiconductors," IEEE Trans. Electron Devices, vol. 48, pp. 567-571, Mar. 2001.

[11] M. Akita, S. Kishimoto, and T. Mizutani, "High-frequency measurements of AlGaN/GaN HEMTs at high temperatures," IEEE Electron Device Lett., vol. 22, pp. 376-377, Aug. 2001.

[12] T.-H. Yu and K. F. Brennan, "Theoretical study of the two-dimensional electron mobility in strained III-nitride heterostructures," J. Appl. Phys., vol. 89, pp. 3827-3834, 2001.

[13] S. C. Binari, K. Ikossi, J. A. Roussos, W. Kruppa, D. Park, H. B. Dietrich, D. D. Koleske, A. E. Wickenden, and R. L. Henry, "Trapping effects and microwave power performance in AlGaN/GaN HEMTs," IEEE Trans. Electron Devices, vol. 48, pp. 465-470, Mar. 2001.

[14] R. Vetury, N. Q. Zhang, S. Keller, and U. K. Mishra, "The impact of surface states on the DC and RF characteristics of AlGaN/GaN HFETs," IEEE Trans. Electron Devices, vol. 48, pp. 560-566, Mar. 2001.

[15] M. Shur, GaAs Devices and Circuits. New York: Plenum, 1987, pp. 369-372.

[16] J. Kuzmik, T. Lalinsky, and Z. Mozolova, "DC performance of shortchannel ion-implanted GaAs MESFETs (the role of gate length shortening)," Solid-State Electron., vol. 33, p. 1223, 1990.

[17] J. W. Johnson, J. Han, A. B. Baca, R. D. Briggs, R. J. Shul, J. R. Wendt, C. Monier, F. Ren, B. Luo, S. N. G. Chu, D. Tsvetkov, V. Dimitriev, and S. J. Pearton, "Comparison of AlGaN/GaN high electron mobility transistors grown on AlN/SiC templates or sapphire," Solid-State Electron., vol. 46, pp. 513-523, 2002.

[18] J. V. DiLorenzo and D. D. Khandelwal, GaAs FET Principles and Technology. $\quad$ Norwood, MA: Artech House, 1982, pp. 308-347. 